\title{
Adaptive Optics for Optical Coherence Tomography in Retinal Imaging: A Reflection on Past and Future Developments
}

\author{
Barry CENSE and Isamuddin IBRAHIM \\ Utsunomiya University Center for Optical Research \& Education, 7-1-2 Yoto, Utsunomiya-city, Tochigi 321-8585
}

(Received August 21, 2013)

\begin{abstract}
OCT is a non-invasive technique for three-dimensional imaging at high axial resolution. Due to aberrations in the eye, OCT systems for retinal imaging have a limited lateral resolution. The lateral resolution can be increased by using adaptive optics (AO) to correct for static and dynamic wavefront aberrations. The combination of these two technologies results in high quality AO-OCT images to image photoreceptors, capillaries and nerve fiber bundles, which can help retinal specialists to better understand and diagnose retinal diseases. Here we review the basic principles of AO-OCT and discuss future opportunities for the technology.
\end{abstract}

Key Words: Optical coherence tomography, Adaptive optics

\section{Introduction}

Vision loss starts at the level of a single cell in the retina (the retina is the back of the eye). The loss of retinal cells is irreversible, making early detection of retinal diseases crucial for prevention of vision loss. The three main modalities for highresolution non invasive retinal imaging are confocal scanning laser ophthalmoscopy (SLO), fundus camera imaging, ${ }^{2)}$ and optical coherence tomography (OCT). ${ }^{3}$ The fundus camera provides no depth sectioning capability, while SLO offers some confocality to depth section tissue, such as imaging the surface of the retina (where the nerve fiber bundles are located) or the photoreceptor mosaic. Only OCT provides high axial resolution for three dimensional imaging. Standard OCT systems for retinal imaging suffer from aberration in the eye and its relatively low lateral resolution prevents imaging at a cellular level. The lateral resolution can be improved with adaptive optics (AO) ${ }^{4-6)}$ This combination gives high quality

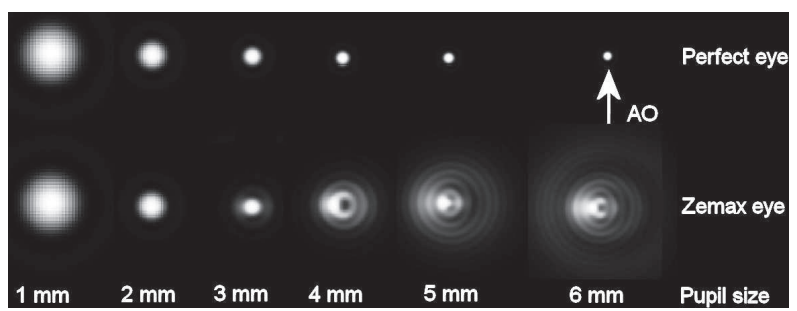

Fig. 1 PSFs at the retina for different pupil sizes in a perfect eye and a human eye modeled in Zemax. A large pupil size combined with AO allows for correction of aberrations to produce a smaller PSF and higher quality retinal images. three dimensional OCT images of the human retina to give ophthalmologists a better understanding of blinding retinal diseases such as glaucoma and age-related macular degeneration (AMD)..$^{7-12)}$

To illustrate the benefit of adaptive optics for retinal imaging we simulated the point spread function as obtained at the retina for different beam sizes in the ray-tracing software package Zemax, using a model eye designed by Liou and Brennan ${ }^{13)}$ and adapted to Zemax by Tocci, ${ }^{\dagger 1}$ after an idea by Roorda. $^{\dagger 2,14)}$ In this simulation, the point spread function (PSF) was modeled for six different beam sizes, with and without use of adaptive optics (see Fig. 1).

At a beam diameter smaller than $\sim 1.2 \mathrm{~mm}$, a correction of defocus is sufficient to achieve diffraction-limited performance. ${ }^{15-17)}$ At beam diameters larger than $1.2 \mathrm{~mm}$, higher-order aberrations can become dominant in human eyes. The Zemax model eye still gives a diffraction-limited spot size when a $2 \mathrm{~mm}$ beam size is used. For most human subjects and a 2 $\mathrm{mm}$ beam size, the spot size will however not be diffraction limited. ${ }^{16)}$ Apparently, the Zemax model eye does not have any defocus or astigmatism aberrations. Even at $3 \mathrm{~mm}$ the Zemax eye gives a spot size core diameter that is smaller than the core size for the $2 \mathrm{~mm}$ pupil, but in comparison to the AOcorrected spot, one can see the effects of aberrations. At 4 $\mathrm{mm}$, the effects of (high order) aberrations are clearly visible, and at 5 and $6 \mathrm{~mm}$ aberrations severely deteriorate the spot quality. The benefit of AO should be evident from these images: with increasing beam size the AO-corrected spot size decreases. A retinal image obtained with AO-OCT is a convolution of the PSF with the structures in the retina, and the 6 $\mathrm{mm}$ beam size with $\mathrm{AO}$ will therefore provide the image with the highest resolution and highest image quality. The pupil of

\footnotetext{
${ }^{\dagger 1} \mathrm{M}$. Tocci: How to model the human eye in ZEMAX. ZeMax knowledge base. Available from: http://www.contrastoptical.com/wp/wp-content/ uploads/2013/05/CODE_Eye_article.pdf.

${ }^{\dagger 2}$ A. Roorda: Review of Optics. Available from: http://voi.opt.uh.edu/1_Roorda_OpticPrinciples.pdf.
} 
the eye will have to be dilated with pharmaceuticals to achieve a $6 \mathrm{~mm}$ aperture size. One should keep in mind that there are individuals with eye optics that have only very small aberrations, even when larger beam sizes are used. An OCT system with a large aperture without adaptive optics may give high resolution images for these particular eyes, but such a machine will be useless in a clinic, where patients with lesser quality optics are imaged.

Recently, there have been multiple reviews on the application of adaptive optics for retinal imaging purposes, ${ }^{7-12,14,18)}$ with several of them dedicated to adaptive optics and OCT. We therefore limit this review to the basic principles of OCT and $\mathrm{AO}$ systems, along with results obtained with AO-OCT after the publication of those review papers. We will also discuss current and future developments for AO-OCT, and reflect on what is required to bring the technology from the laboratory to the clinic.

\section{Optical Coherence Tomography and Adaptive Optics}

We will first introduce OCT, followed by an introduction of AO technology. The two most popular methodologies for OCT are spectral-domain OCT (SD-OCT) and swept-source OCT (SS-OCT). In SD-OCT, near infra red light from a broadband light source with low temporal coherence and high spatial coherence, such as a superluminescent diode (SLD) or a Ti:Sapphire laser, is sent into a Michelson interferometer where it is split by a beam splitter towards a sample and a reference arm (see Fig. 2). The power that is used for retinal imaging is kept well below the standards for safe use of (laser) light. ${ }^{19)}$ Single-mode fiber couplers are often used for this purpose. In the sample arm, the retina is scanned with an XYraster scanner and several lenses. ${ }^{20,21)}$ The optical path lengths of sample and reference arms are matched, and the optical chromatic dispersion in the interferometer is kept at a minimum by matching the dispersion in sample and reference arm, to avoid blurring of images. ${ }^{22}$ In the reference arm, a combination of lenses and mirror returns light to the beam splitter, where light recombines with light returning from the retina. One can use a variable neutral density filter to ensure that the reference signal dominates the sample signal without detector saturation. Polarization controllers are used to match the polarization state of sample and reference arm, for an optimal interference signal. The interference is detected with a highspeed spectrometer, consisting of a collimator, a (transmission) grating, a lens and a line scan camera (Fig. 2).

The data is mapped from detector space to k-space and Fourier-transformed to retrieve depth scans (A-scans). ${ }^{23,24)}$

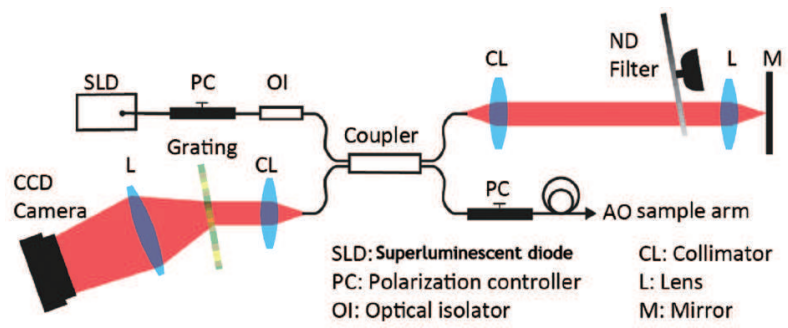

Fig. 2 Typical setup for SD-OCT. The CCD camera is connected to a frame grabber and computer for data offload and processing. In standard retinal imaging, typically an SLD with a center wavelength of $840 \mathrm{~nm}$ and a bandwidth of $50 \mathrm{~nm}$ (full width at half maximum) is used, giving a coherence length in the retina of approximately $6 \mu \mathrm{m}$.
The axial resolution of OCT can be improved by using broader bandwidth light sources. ${ }^{25,26)}$ SS-OCT works somewhat differently, in a sense that it uses a light source with a very narrow bandwidth filter that is quickly swept through a spectrum. ${ }^{27-29)}$ While SD-OCT systems use a linescan camera in the detection arm, SS-OCT systems use photodiodes in balanced detection mode. The detected signal is often acquired in k-space and Fourier-transformed to extract A-scans. The sampling at very narrow bandwidth allows for a more accurate measurement of the high frequency spectral modulations involved with reflections that occur at a larger optical path length difference. SS-OCT therefore provides a wider imaging window than SD-OCT, and it will not suffer as much from sensitivity decay with depth. Most commercial OCT systems however are still based on SD-OCT technology. More information about OCT technology for retinal imaging and in other applications can be found in various reviews. ${ }^{30-33}$

Adaptive optics was first used in the military in groundbased telescopes for imaging and tracking of spy satellites, and later in astronomy and vision science. ${ }^{7.34-36)}$ In general, an AO system consists of a wavefront sensor, a control system and a wavefront corrector. A wavefront sensor measures wavefront deviations and for retinal imaging applications the Shack-Hartmann sensor is by far the most popular option. It contains a two-dimensional lenslet array with micro lenses that share the same diameter and focal length. An incoming beam is divided into individual beams by each of the lenslets and spots are recorded in the focal plane with a camera. The control system uses this information to calculate the wavefront. A higher lenslet density ensures a higher wavefront measurement accuracy, but also limits the dynamic range over which the sensor can be used, because spots will overlap at moderate aberrations. When fewer lenslets are used, the wavefront is sampled more coarsely, but aberrations with a high magnitude can be measured without an overlap of spots. For retinal imaging, the deformable mirror (DM) is the most popular option for wavefront correction. ${ }^{37}$ It can change shape in response to input commands. Another option for wavefront correction in AO-OCT is a spatial light modulator (SLM). In contrast to the DM, the SLM is sensitive to chromatic dispersion and to the input polarization state, which makes the SLM less suitable for OCT, where broadband sources are used and light returning from the retina will have an unknown polarization state, due to birefringence in the retina and cornea (the cornea is the front of the eye).

In an AO design so-called pupil planes are conjugated with each other, meaning that the pupil of the human eye is imaged onto the galvanometer raster scanners, the DM and the ShackHartmann wavefront sensor. ${ }^{7)}$ This allows for a stable beam in each of the pupil planes, so that the wavefront can be accurately determined and correction is not affected by a shifting beam, while the retina is raster scanned. By using telescopes with different magnifications, the beam size can be matched to the aperture size of the galvanometer raster scanners, DM and pupil of the eye. Retinal planes are also conjugated, with the retina imaged onto the single mode fiber of the OCT system. When pupil planes are not carefully conjugated, the beam will shift over the cornea during scanning, so that different aberrations occur, which are often impossible to compensate in real time. The conjugation of pupil planes require several optical telescopes, with each of the telescopes inducing optical loss and aberrations. When spherical mirrors are used in a telescope configuration, the beam will hit each mirror at an angle, thereby inducing astigmatism. One can have the adaptive optics system correct for these aberrations, but this takes up DM 
stroke, which limits the dynamic range of the AO system for compensation of defocus aberrations. Another solution is to bring two sets of telescopes out of the plane, so that the astigmatism induced by one set of telescopes is compensated by the other set. ${ }^{38)}$ Several groups have worked on this concept in AO-SLO and AO-OCT devices, to minimize the astigmatism in the system. ${ }^{39-42}$ Toroidal mirrors have a similar effect as the previous solutions, with a more straightforward system alignment, as all components are oriented in the same plane. ${ }^{43)}$ Lenses can also be used instead of spherical mirrors, with the advantage that they can be used oriented perpendicular to the beam. $^{44)}$

The first AO-OCT images were demonstrated 10 years ago by Miller $e$ al $^{4}{ }^{4}$ Looking back at AO-OCT technology development in the past ten years, data acquisition speeds were improved and systems were outfitted with ultra broadband light sources. DMs with higher fidelity and larger strokes were introduced. Furthermore, combinations with other modalities such as an AO-SLO or retinal tracker and sophisticated scan patterns lead to images with a higher resolution and higher stability. Small retinal features such as the cone photoreceptor mosaic, retinal nerve fiber bundles, foveal capillaries and the choriocapillaris have been imaged with AO-OCT. ${ }^{7-11}$ )

\section{Other opportunities for AO-OCT technology}

Given that AO-OCT provides high quality images at a resolution five times higher than standard $\mathrm{OCT}^{45)}$ one may wonder why the technology has not progressed from the laboratory to the clinic, where it can be used on patients. What needs to be done in order to make AO-OCT a modality that is (commercially) available to retinal specialists, so that it can be used routinely in the clinic? If we look beyond resolution, what other opportunities does AO offer for OCT?

AO-OCT technology is expensive. Since components such as the DM and Shack-Hartmann sensor are not widely used outside the laboratory, they are very costly. If these components were to be used on a larger scale for other purposes, similar to the low-cost but high-tech (fiber) telecom products that made SD-OCT a commercial success, a lower bill of materials could be expected. In the current market, the cost of an AO-OCT system surpasses the cost of a standard OCT system several times. Earlier, we discounted SLMs because of chromatic aberration and its sensitivity to the polarization state of light, but SLMs are widely used, and their relatively low cost makes them more interesting for commercial applications. Other optical and opto-mechanical devices, such as high-quality spherical mirrors and multi-axis mirror mounts are not cheap either. Because of the conjugation of pupil planes, an AO-OCT system takes up much space. The alignment of optical components is tedious and requires experienced personnel, who also have to maintain the system, as optical components are likely to become misaligned over time. These factors make the technology less than ideal for a clinical application.

Other issues that make AO-OCT less suitable for a clinical environment are related to physics. When a larger aperture is used, more photons can be collected from the diffuse reflection in the retina, which should result in a higher sensitivity. Traditional AO-OCT systems have only demonstrated moderate gains in sensitivity, which may be attributed to the large number of optical surfaces with $<98 \%$ reflectivity that are used to conjugate retinal and pupil planes. At for instance an average optical loss per element of $3.5 \%$, a total loss of $50 \%$ is achieved in single pass, corresponding to a $3 \mathrm{~dB}$ loss in sensitivity. ${ }^{46}$

Taking data with an AO-OCT system is difficult. The patient's eye has to be perfectly aligned, so that it conjugates well with the other pupil planes, and a Shack-Hartmann image has to be obtained that is free of artifacts, to retrieve reliable wavefront data. As experienced AO-OCT users will know, most young healthy subjects will provide good wavefront data and easy correction, while older eyes are more challenging. In particular, drier corneas will make AO-OCT correction difficult and shifting eyes of subjects with poor fixation are pretty much impossible to image. Non-common path errors are another issue, as correction in AO-OCT is optimized for the Shack-Hartmann sensor channel wavefront, while the end user is in fact more interested in the image quality in the OCT channel (see Fig. 3). A slight difference in the optics in the OCT channel in comparison to the wavefront measurement channel may result in an OCT signal loss. The elimination of the Shack-Hartmann sensor in a sensor-less AO operation would make AO-OCT more affordable, and perhaps easier to use at a better performance, since non-common path errors are avoided. $^{47, \uparrow 3)}$

Measured from the inner limiting membrane to the retinal pigment epithelium at $3^{\circ}$ eccentricity, a human retina typically measures 300-350 $\mu \mathrm{m}$. In these layers, OCT provides the best contrast for imaging. A consequence of the $3.5 \mu \mathrm{m}$ lateral resolution is a $\sim 50 \mu \mathrm{m}$ Rayleigh length, forcing the operator to focus the AO-OCT system either at the inner- or outer retina. ${ }^{48)}$ By applying active phase modulation of the AO pupil, the depth of focus can be extended, but the spot size at the ret-

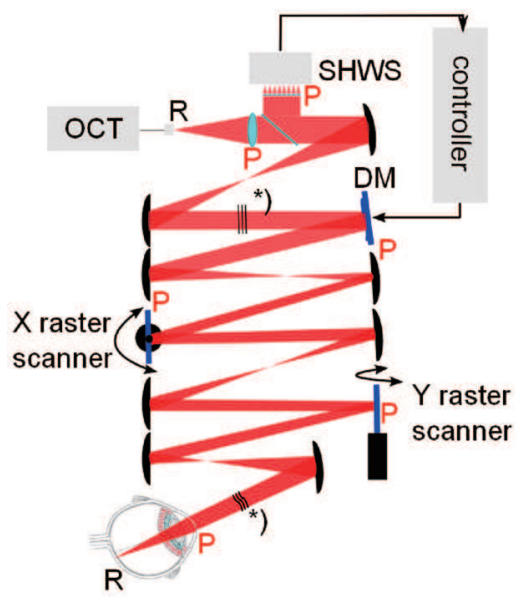

Fig. 3 A typical AO closed-loop design for retinal imaging, with spherical mirrors conjugating pupil planes (" $P$ "); " $R$ " indicates a retinal plane. Light returning from the eye is split between a wavefront sensor (SHWS) channel and OCT channel. The wavefront is computed from the SHWS image, and the control system determines the shape of the deformable mirror (DM) to correct for aberrations. When the wavefront is perfectly flat, the retina can be imaged at the diffraction limit. The lines near the two asterisks model the wavefront shape at different locations in the system.

${ }^{\dagger 3}$ S. Bonora, R. Zawadzki, G. Naletto, U. Bortolozzo, and S. Residori: Devices and Techniques for Sensorless Adaptive Optics. Available from: http://cdn.intechopen.com/pdfs/41393/InTech-Devices_and_techniques_for_sensorless_adaptive_optics.pdf. 
ina increases. ${ }^{49)}$ Moreover, the isoplanatic patch (retinal area over which the AO system is diffraction-limited) of AO systems with a $6 \mathrm{~mm}$ beam is limited to less than $2^{\circ}$ (less than $600 \mu \mathrm{m}){ }^{50)}$ which makes surveying of large retinal areas for clinical applications more difficult. At a $3.5 \mu \mathrm{m}$ lateral resolution, such a survey also requires a long scanning time. For instance, imaging a $10^{\circ}$ by $10^{\circ}(3 \mathrm{~mm}$ by $3 \mathrm{~mm})$ retinal area with $\mathrm{AO}-\mathrm{OCT}$ at 70,000 A-scans/s would require $10.5 \mathrm{~s}$. Keeping Nyquist theorem in mind, $42 \mathrm{~s}$ are needed to correctly sample at $3.5 \mu \mathrm{m}$ resolution. At this acquisition time, the image would suffer from too much eye motion. A retinal tracker may provide an answer here, ${ }^{51)}$ but an acquisition time of $42 \mathrm{~s}$ will be too long for the average patient. Moreover, the $10^{\circ}$ by $10^{\circ}$ field of view (FOV) requires 25 wavefront measurements and corrections, due to the $2^{\circ}$ isoplanatic patch. Very fast wavefront sensors, correctors and controllers, similar to the ones used in astronomy, may be able to correct these during a scan, but so far this has not been demonstrated. In this example we used a $10^{\circ}$ by $10^{\circ} \mathrm{FOV}$ but (laboratory) OCT systems routinely image a $30^{\circ}$ by $30^{\circ} \mathrm{FOV}$. The isoplanatic patch of a standard OCT system with a $1.2 \mathrm{~mm}$ beam size at the cornea covers such a large FOV, but its spot and speckle size is five times larger than the diffraction-limited spot size of an AOOCT system. ${ }^{45)}$

This brings us to a general thought: in the past ten years the AO vision science community has focused efforts on achieving the highest possible lateral resolution. While such a high lateral resolution nicely complements the confocality of AOSLO systems, it is highly detrimental to the natural depth-sectioning capability of OCT, because the short Rayleigh length blurs out half of the OCT image. And, while AO technology has been extensively used to image cone photoreceptors (and rod photoreceptors), ${ }^{41)}$ there are only so many retinal diseases that depend on photoreceptor imaging. Standard OCT images and AO-OCT images suffer from speckle noise, a grainy pattern that blocks clinicians from getting a good view on small retinal features, even if images are obtained at high resolution. Recently, commercial OCT instruments have been using speckle averaging methods, by acquiring and averaging closely spaced scans, to reduce speckle contrast. AO-OCT technology can contribute to a solution and this will be addressed at the end of this section.

By reducing the beam size of an adaptive optics system, a sweet spot may be found for clinical AO-OCT imaging, using an aperture size of around $3.4 \mathrm{~mm} .{ }^{52}$ Technically, this would not be an AO-OCT system, but its benefits for clinical imaging speak for itself. Its longer Rayleigh length allows imaging of the full depth of the retina with OCT and it will have an isoplanatic patch size of $10^{\circ}$ by $10^{\circ}$ that makes clinical applications practical at a lateral resolution that is considerably higher than the resolution of a commercial OCT system. At this resolution and at an acquisition speed of 70,000 A-scans/s, the retina can be scanned in $2.3 \mathrm{~s}$. If we do consider Nyquist theorem, the acquisition time approaches $10 \mathrm{~s}$. One potential strategy would be to image a volume coarsely in $2.3 \mathrm{~s}$ (but still at a higher resolution than a standard OCT system), and then focus on areas of interest at the highest sampling density, thereby keeping the acquisition time to a minimum, to limit the effects of eye motion. Again, a retinal tracker would be beneficial.

Another advantage of a $3.4 \mathrm{~mm}$ beam is that a lesser quality wavefront corrector can be used, since the correction of aberrations that are induced in a smaller aperture are less demanding. ${ }^{16,37)}$ For instance, a $6.0 \mathrm{~mm}$ beam requires correction up to the 6 th or 7 th radial order, while correction of a $3.0 \mathrm{~mm}$ beam only requires correction up to the 4 th radial order (which includes defocus, oblique and vertical astigmatism, vertical and horizontal coma, vertical and oblique trefoil and spherical aberration). ${ }^{16)}$ The $6.0 \mathrm{~mm}$ system requires a wavefront corrector with both high fidelity (i.e. many actuators) and high stroke, while the latter can be performed with a corrector that has fewer elements and a smaller stroke, meaning that a much more affordable DM can be used instead. This idea can be pushed further. A $3.4 \mathrm{~mm}$ beam system would still need a relatively sophisticated wavefront corrector, but the aberrations for a $\sim 2 \mathrm{~mm}$ aperture could be corrected with a Badal optometer (an ophthalmic lens that is shifted perpendicular to the beam direction to correct for defocus) and a set of astigmatic lenses to correct for astigmatism. As shown in Fig. 1, a corrected $2 \mathrm{~mm}$ beam would indeed give an almost 2 times spot size reduction in comparison to a standard $1.2 \mathrm{~mm}$ aperture, at minimal extra costs. If we were to assume that the light intensity returning from the retina is evenly distributed (which makes the calculation easier but is an incorrect assumption because of the way the light is scattered from the retina, think Stiles-Crawford effect, ${ }^{53)}$ among others), a doubling of the aperture size would result in a four times increase in sensitivity, or $6 \mathrm{~dB}$. Short-term, a lens-based system with a $\sim 2 \mathrm{~mm}$ beam may be the fastest way to use AO technology in a clinical environment.

Since hospitals typically do not have much space for an optical table-sized AO-OCT system, its size should preferably be reduced to the size of a standard OCT system. One can consider bringing telescopes out of the plane, not only to reduce system astigmatism, but also to save space. Our $3.4 \mathrm{~mm}$ design resulted in a prototype with a $25 \mathrm{~cm}$ by $50 \mathrm{~cm}$ footprint, see Fig. $4{ }^{52)}$ The size of this system only barely surpasses the size of a typical commercial OCT system.

The system in Fig. 4 was used to image the retina of a patient with macular degeneration. ${ }^{52)}$ Volumetric data sets were

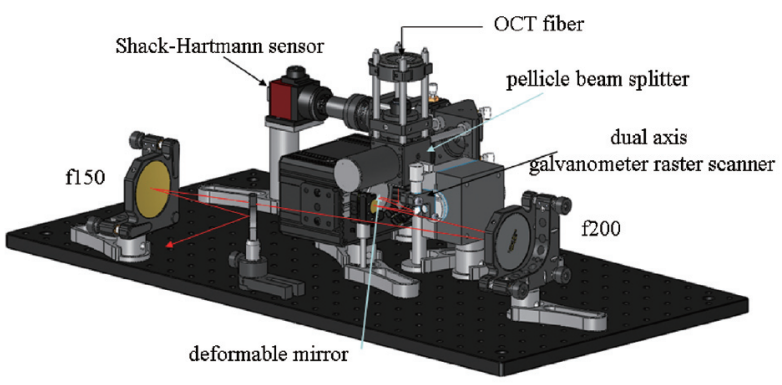

Fig. 4 Design in SolidWorks of an OCT system with a $3.4 \mathrm{~mm}$ aperture and a $25 \mathrm{~cm}$ by $50 \mathrm{~cm}$ footprint.

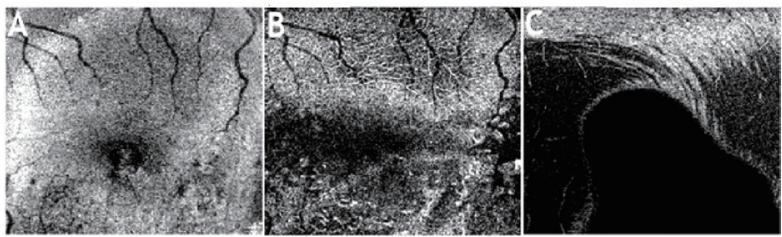

Fig. $58^{\circ}$ by $8^{\circ}$ en face OCT images simultaneously obtained in $\sim 3.6 \mathrm{~s}$ with a $3.4 \mathrm{~mm}$ beam size and adaptive optics from a patient with macular degeneration, showing the diseased tissue (bottom of the images) and healthier tissue (top of the images). Image A was collected near the photoreceptor layer, image B near the outer plexiform layer and image $\mathrm{C}$ through the nerve fiber layer. 
collected over $8^{\circ}$ by $8^{\circ}$, and en face C-scans were generated to demonstrate the large Rayleigh range of this system, with both the fine structures in the photoreceptor mosaic and retinal nerve fiber bundles visible (see Fig. 5), and an almost 3 times reduction in speckle size. ${ }^{52)}$ When the system operates in the diffraction-limit, a spot size of $7.0 \pm 0.4 \mu \mathrm{m}$ is achieved.

Another direction may lie in the use of post-processing algorithms for aberration correction. Recently, two groups have shown interesting results on non-retinal tissue. Kumar et al. used a phase-sensitive full field OCT system without adaptive optics to image tissue and remove aberrations. ${ }^{54)}$ Ahmad et al. used a somewhat similar approach. ${ }^{55)}$ In a shifting human eye and a fiber-based OCT system (which is not a full field imaging modality but a point imaging approach), it will be much harder to preserve the phase, making aberration correction in post-processing considerably more difficult. Still, as the acquisition speed of OCT systems is steadily increasing, the brute force of a high acquisition speed may be used to acquire high-quality data. To illustrate the potential of high-speed acquisition for OCT, Klein et al. recently demonstrated the power of their joint aperture detection: using four OCT beams simultaneously in a system without AO, they were able to reduce speckle contrast in retinal OCT images, thereby achieving a much higher image quality compared to averaged standard OCT images. These images did not show the gain in lateral resolution that is obtained with AO-OCT, but the improvement in image quality due to speckle averaging was obvious.

Similarly, by acquiring three tomograms spaced $\sim 6 \mu \mathrm{m}$ from each other with a $3.4 \mathrm{~mm}$ system, 9 independent point spread functions are obtained within the point spread function of a standard OCT system $(\sim 20 \mu \mathrm{m})$, resulting in a three times reduction in speckle contrast. Measurements in the field will have to prove which attribute clinicians prefer most: an increase in resolution, an improved speckle contrast reduction, an increase in sensitivity or the versatility to have these capabilities in one device.

\section{Summary}

The combination of AO and OCT results in high quality images to image photoreceptors, capillaries and nerve fiber bundles, which can help retinal specialists to better understand and diagnose retinal diseases. We gave an overview of the technology involved in standard AO-OCT and discussed the progress in technology. We also peeked into the distant future, and discussed the opportunities that AO offers for OCT.

\section{Acknowledgments}

The authors thank Dr. Robert Zawadzki from UC Davis and Hans Verstraete from the Technical University Delft for helpful discussions and former student Kenta Sudo for his contributions to the $3.4 \mathrm{~mm}$ OCT project.

\section{References}

1) R. H. Webb, G. W. Hughes, and O. Pomerantzeff: Appl. Opt. 19 (1980) 2991.

2) L. A. Yannuzzi, M.D. Ober, J. S. Slakter, R. F. Spaide, Y. L. Fisher, R. W. Flower, and R. Rosen: Am. J. Ophthalmol. 137 (2004) 511.

3) D. Huang, E. A. Swanson, C. P. Lin, J. S. Schuman, W. G. Stinson, W. Chang, M. R. Hee, T. Flotte, K. Gregory, C. A. Puliafito, et al.: Science 254 (1991) 1178.

4) D. T. Miller, J. Qu, R. S. Jonnal, and K. E. Thorn: Proc. Biomedi- cal Optics 2003, 2003 (International Society for Optics and Photonics) p. 65.

5) R. J. Zawadzki, S. M. Jones, S. S. Olivier, M. T. Zhao, B. A. Bower, J. A. Izatt, S. Choi, S. Laut, and J. S. Werner: Opt. Express 13 (2005) 8532.

6) Y. Zhang, J. T. Rha, R. S. Jonnal, and D. T. Miller: Opt. Express 13 (2005) 4792

7) J. Porter, H. Queener, J. Lin, K. E. Thorn, and A. Awwal: Adaptive Optics for Vision Science: Principles, Practices, Design and Applications. (Wiley, New York, 2006).

8) M. Pircher and R. J. Zawadzki: Expert Rev. Ophthalmol. 2 (2007) 1019.

9) P. Godara, A. M. Dubis, A. Roorda, J. L. Duncan, and J. Carroll: Optometry and vision science: official publication of the American Academy of Optometry 87 (2010) 930.

10) D. R. Williams: Vision Research 51 (2011) 1379.

11) D. T. Miller, O. P. Kocaoglu, Q. Wang, and S. Lee: Eye 25 (2011) 321.

12) J. Carroll, D. B. Kay, D. Scoles, A. Dubra, and M. Lombardo: Current Eye Research 38 (2013) 709.

13) H. L. Liou and N. A. Brennan: J. Opt. Soc. Am. A 14 (1997) 1684.

14) A. Roorda: Journal of vision 11 (2011) 6.

15) F. W. Campbell and D. G. Green: J. Physiology-London 181 (1965) 576.

16) L. N. Thibos, X. Hong, A. Bradley, and X. Cheng: J. Opt. Soc. Am. A 19 (2002) 2329.

17) E. J. Fernandez and W. Drexler: Opt. Express 13 (2005) 8184

18) A. Roorda: Optometry and Vision Science 87 (2010) 260

19) F. C. Delori, R. H. Webb, and D. H. Sliney: J. Opt. Soc. Am. A 24 (2007) 1250

20) M. R. Hee, J. A. Izatt, E. A. Swanson, D. Huang, J. S. Schuman, C. P. Lin, C. A. Puliafito, and J. G. Fujimoto: Archives of Ophthalmology 113 (1995) 325.

21) B. Cense, T. C. Chen, B. H. Park, M. C. Pierce, and J. F. de Boer: J. Biomed. Opt. 9 (2004) 121.

22) W. Drexler, U. Morgner, R. K. Ghanta, F. X. Kartner, J. S. Schuman, and J. G. Fujimoto: Nature Medicine 7 (2001) 502.

23) N. Nassif, B. Cense, B. H. Park, S. H. Yun, T. C. Chen, B. E. Bouma, G. J. Tearney, and J. F. de Boer: Opt. Lett. 29 (2004) 480.

24) N. A. Nassif, B. Cense, B. H. Park, M. C. Pierce, S. H. Yun, B. E. Bouma, G. J. Tearney, T. C. Chen, and J. F. de Boer: Opt. Express 12 (2004) 367

25) M. Wojtkowski, V. J. Srinivasan, T. H. Ko, J. G. Fujimoto, A. Kowalczyk, and J. S. Duker: Opt. Express 12 (2004) 2404.

26) B. Cense, N. A. Nassif, T. C. Chen, M. C. Pierce, S. H. Yun, B. H. Park, B. E. Bouma, G. J. Tearney, and J. F. de Boer: Opt. Express 12 (2004) 13.

27) B. Golubovic, B. E. Bouma, G. J. Tearney, and J. G. Fujimoto: Opt. Lett. 22 (1997) 1704.

28) M. A. Choma, M. V. Sarunic, C. H. Yang, and J. A. Izatt: Opt. Express 11 (2003) 2183.

29) S. H. Yun, G. J. Tearney, J. F. de Boer, N. Iftimia, and B. E. Bouma: Opt. Express 11 (2003) 2953.

30) B. E. Bouma and G. J. Tearney (eds.): Handbook of Optical Coherence Tomography (Marcel Dekker, Inc., New York, 2002).

31) A. F. Fercher, W. Drexler, C. K. Hitzenberger, and T. Lasser: Reports on Progress in Physics 66 (2003) 239.

32) M. E. van Velthoven, D. J. Faber, F. D. Verbraak, T. G. van Leeuwen, and M. D. de Smet: Progress in Retinal and Eye Research 26 (2007) 57.

33) W. Drexler and J. G. Fujimoto: Optical coherence tomography: technology and applications (Springer, 2008).

34) H. W. Babcock: Pub. Astronomical Soc. Pacific 65 (1953) 229.

35) J. W. Hardy: Adaptive Optics for Astronomical Telescopes (Oxford University Press, 1998).

36) R. Tyson: Principles of Adaptive Optics (CRC Press, 2010).

37) E. Dalimier and C. Dainty: Opt. Express 13 (2005) 4275.

38) K. Araki: Opt. Rev. 12 (2005) 219.

39) S. A. Burns, R. Tumbar, A. E. Elsner, D. Ferguson, and D. X. Hammer: J. Opt. Soc. Am. A 24 (2007) 1313.

40) K. Kurokawa, D. Tamada, S. Makita, and Y. Yasuno: Opt. Express 18 (2010) 1406

41) A. Dubra, Y. Sulai, J. L. Norris, R. F. Cooper, A. M. Dubis, D. R. 
Williams, and J. Carroll: Biomedical Opt. Express 2 (2011) 1864.

42) S. H. Lee, J. S. Werner, and R. J. Zawadzki: Proc. SPIE BiOS, 2013 (International Society for Optics and Photonics) p. 856722.

43) Z. Liu, O. P. Kocaoglu, and D. T. Miller: Biomed. Opt. Express 4 (2013) 3007

44) F. Felberer, J. S. Kroisamer, C. K. Hitzenberger, and M. Pircher: Opt. Express 20 (2012) 17297.

45) B. Cense, W. Gao, J. M. Brown, S. M. Jones, R. S. Jonnal, M. Mujat, B. H. Park, J. F. de Boer, and D. T. Miller: Opt. Express 17 (2009) 21634.

46) B. Cense, E. Koperda, J. M. Brown, O. P. Kocaoglu, W. H. Gao, R. S. Jonnal, and D. T. Miller: Opt. Express 17 (2009) 4095.

47) H. Hofer, N. Sredar, H. Queener, C. Li, and J. Porter: Opt. Express 19 (2011) 14160.

48) O. P. Kocaoglu, B. Cense, R. S. Jonnal, Q. Wang, S. Lee, W. Gao, and D. T. Miller: Vision Research 51 (2011) 1835.
49) K. Sasaki, K. Kurokawa, S. Makita, and Y. Yasuno: Biomedical Opt. Express 3 (2012) 2353.

50) P. Bedggood, M. Daaboul, R. Ashman, G. Smith, and A. Metha: J. Biomed. Opt. 13 (2008) 024008.

51) R. D. Ferguson, D. X. Hammer, L. A. Paunescu, S. Beaton, and J. S. Schuman: Opt. Lett. 29 (2004) 2139.

52) K. Sudo and B. Cense: Proc. SPIE BiOS, 2013 (International Society for Optics and Photonics) p. 85671W.

53) W. Gao, B. Cense, Y. Zhang, R. S. Jonnal, and D. T. Miller: Opt. Express 16 (2008) 6486.

54) A. Kumar, W. Drexler, and R. A. Leitgeb: Opt. Express 21 (2013) 10850 .

55) A. Ahmad, N. D. Shemonski, S. G. Adie, H.-S. Kim, W.-M. W. Hwu, P. S. Carney, and S. A. Boppart: Nature Photon. 7 (2013) 444. 